\title{
Power-Estimation for on-Chip VLSI Distributed RLC Global Interconnect using Model Order Reduction Technique
}

\author{
R. Kar \\ VLSI Laboratory \\ Department of ECE \\ NIT Durgapur-713209
}

\author{
V. Maheshwari \\ VLSI Laboratory \\ Department of ECE \\ NIT Durgapur-713209
}

\author{
Ashis K. mal \\ VLSI Laboratory \\ Department of ECE \\ NIT Durgapur-713209
}

\author{
A.K.Bhattacharjee \\ VLSI Laboratory \\ Department of ECE \\ NIT Durgapur-713209
}

\begin{abstract}
Power is increasingly becoming the bottleneck for the design of high performance VLSI circuits. It is essential to analyze how the various components of power are likely to scale in the future, thereby identifying the key problematic areas. While most analyses focus on the timing aspects of interconnects, power consumption is also important. In this paper, the power distribution estimation of interconnects is studied using a reducedorder model [1]. The relation between power consumption and the poles and residues of a transfer function is derived, and an appropriate driver model is developed, allowing power consumption to be computed efficiently.
\end{abstract}

\section{Categories and Subject Descriptors}

B.7.2 [Integrated Circuits]: Design Aids - Simulation;

\section{General Terms}

Algorithms, Design, Theory

\section{Keywords}

Power estimation, Model Order Reduction, RCL Interconnect, Moment matching

\section{INTRODUCTION}

As the scale of process technologies steadily shrinks and the size of designs increases, interconnects have increasing impact on the area, delay, and power consumption of circuits. Over the past decade there have been a number of advances in modeling and the analysis of interconnect that have facilitated the continual advances in design automation for systems of increasing size and frequency. As integrated circuit feature sizes continue to scale well below $0.18 \mu \mathrm{m}$ [2], active device counts are reaching hundreds of millions. Interconnect models must incorporate distributed self and mutual inductance to accurately estimate time delay and crosstalk in a multilevel network for multi- $\mathrm{GHz}$ gigascale integration (GSI) [3]. In addition to interconnect delay, crosstalk noise resulting from capacitive and, more recently investigated, inductive effects [4], [5] between adjacent interconnect lines is also becoming a primary concern for ICs performance and reliability. Furthermore, with present VLSI technology, on-chip interconnects are best modeled as a network of coupled lines the amount of interconnect among the devices tends to grow super linearly with the transistor counts, and the chip area is often limited by the physical interconnect area. Due to these interconnect area limitations, the interconnect dimensions are scaled with the devices whenever possible. In addition, to provide more wiring resources, IC's now accommodate numerous metallization layers, with more to come in the future. These advances in technology that result in scaled, multi-level interconnects may address the wire ability problem, but in the process create problems with signal integrity and interconnect delay. As regards power, the situation is similar in that the portion of power associated with interconnects is increasing. This is an important fact because the conventional design, analysis, and synthesis of VLSI circuits are based on the assumption that gates are the main sources of on-chip power consumption. Furthermore, the power consumed by interconnects results in a phenomenon, called self heating, which reduces electro-migration induced mean time to failure (MTF) [6]. It is shown in [7] that the power distribution analysis on interconnects is feasible in frequency domain using poles and residues. However, high complexity is inevitable when calculating the power dissipation of the whole interconnects since poles and residues of the current flowing through each element have to be calculated. As feature sizes are decreased to deep sub-micrometer dimensions, on-chip interconnect is best modeled as a distributed RLC line. However, unlike the RC model, such a model increases the complexity of interconnects crosstalk noise and its induced delay estimation. Advances in deep sub-micron technology indicate that present and future interconnects might no longer be considered as simply made of RC lines. Thus, RLC interconnect models become a necessity [8]. It therefore appears that, if accurate interconnect delay estimation is to be achieved, modeling interconnect as a distributed RLC line is necessary. In this case, the commonly and generally well-accepted Elmore delay calculation becomes inapplicable to RLC interconnect networks due to their nonmonotonic characteristics induced by inductances [8] [9]. To verify the effects induced by interconnects a combination of extraction and analysis is necessary. Extraction determines the capacitance and the resistance of interconnects, which can then be used to build a circuit model for the analysis of interconnect effects. For analysis (or estimation), extensive studies have been made of the use of model order reduction over the last few years, following the introduction of AWE [9]. Model order reduction is based on approximating the Laplace-domain transfer function of a linear network by a relatively small number of dominant poles and zeros. Such reduced-order models can be used to predict the timedomain or frequency-domain response of the linear network. Power, which inherently involves improper integration, can be derived from the poles and residues of the transfer function, which requires only algebraic computation. When the interconnect is driven by MOSFETs and connected to the gates of MOSFETs, the 
load transistor can be satisfactorily approximated by a capacitor. And we show that the driver transistor can be modeled by a linearregion resistance with sufficient accuracy for power estimation [10]. In this paper, we expand the work presented in [10] by including the effective distributive inductance and derive an analytical delay expression for power distribution of interconnects to be estimated.

\section{BASIC THEORY}

\subsection{Model Order Reduction}

Due to the large number and complex nature of on-chip interconnects; it is impractical to run SPICE-like accurate simulations on an entire IC. If more moments are required for an accurate approximation, moment matching or other order reduction schemes can be used to generate reduced-order dominant pole or zero approximations for the interconnect transfer, admittance, and impedance functions[1]. Model order reduction is a technique that takes a circuit and reduces it to a smaller representation consisting of the dominant poles from the original circuit. There are two approaches to model order reduction: moment matching and matrix approximation [11]. In this section, we outline the method based on moment matching [9]. However, we stress the fact that any kind of model order reduction method can be used as part of the power distribution estimation. All of the efficient moment-based models for interconnect analysis are for linear circuits. The overall behavior and performance of a signal on the interconnect path is strongly dependent upon the nonlinear drivers and loads too. One straightforward way of combining moment-based interconnect models and nonlinear components (e.g. transistors) is to characterize the linear interconnect portion of the circuit by a reduced order multiport (refer to Figure -1) [1].
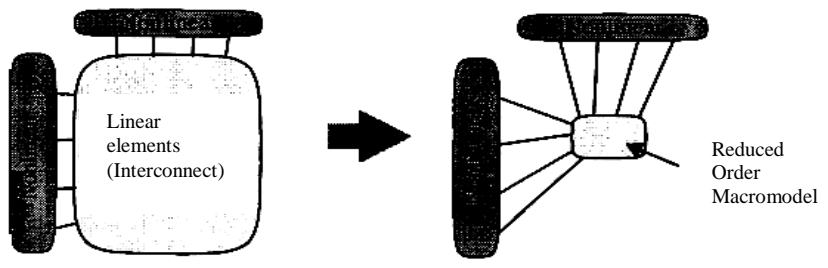

\section{Figure-1 Model Order Reduction of Multiport Interconnect Circuits}

For example, we can approximate the $\mathrm{Y}$ parameters in terms of the dominant poles and zeros. We then combine the reduced order interconnect models and the nonlinear devices in a circuit simulation environment. An important issue for such a simulation is the passivity. We can force the reduced order models to be stable, however, for a stable simulation the reduced order blocks have to be passive as well a lumped, linear, time-invariant circuit can be described by first order differential equations given bellow

$$
\begin{aligned}
& \bar{x}=\mathrm{AX}+\mathrm{bu} \\
& y=c^{T} x+d u
\end{aligned}
$$

Where $\mathrm{x}$ is an $\mathrm{n}$-dimensional state vector, $\mathrm{A}$ is a $n \times n$ matrix, $\mathrm{u}$ is the system's input, $y$ is the output of interest, and d denotes the direct-coupling term. We apply the Laplace transform to (1) \& (2) assuming zero initial conditions and ignoring the term du, which can be treated separately. Then, we obtain

$$
s X=A X+b U \text {, }
$$

$$
Y=c^{T} X
$$

Where $\mathrm{X}, \mathrm{U}$, and $\mathrm{Y}$ denote the Laplace transform of $\mathrm{x}, \mathrm{u}$, and $\mathrm{y}$, respectively. It follows from (2) that the transfer function or the Laplace transform of the impulse response, defined as $H(s)=\frac{Y(s)}{X(s)}$ is

$$
H(s)=c^{T}(s I-A)^{-1} b
$$

Where $\mathrm{I}$ is an identity matrix. If $\mathrm{H}(\mathrm{s})$ has a Taylor series expansion about $s==0$ (i.e. Maclaurin series), then it can be described by

$$
H(s)=\sum_{i=0}^{\infty} m_{i} s^{i}
$$

Substituting (5) into (4) and equating like powers of s, it can be shown that

$$
m_{i}=-c^{T} A^{-i-1} b, \quad \mathrm{i}=0,1,2 \ldots .
$$

The terms mi are related to the moments of the impulse response, denoted by $\mathrm{h}(\mathrm{t})$, because

$$
H(s)=\int_{0}^{\infty} h(t) e^{-s t} d t
$$

Applying a Taylor series expansion of $\mathrm{e}^{\text {-st }}$ about $\mathrm{s}=0$ yields

$$
\begin{aligned}
& H(s)=\int_{0}^{\infty} h(t)\left\{1-s t+\frac{1}{2 !} s^{2} t^{2}-\frac{1}{3 !} s^{3} t^{3}+\ldots .\right\} d t \\
& =\sum_{i=0}^{\infty} \frac{(-1)^{i}}{(i) !} s^{i} \int_{0}^{\infty} t^{i} h(t) d t
\end{aligned}
$$

The $\mathrm{i}^{\text {th }}$ circuit-response moment [9], $\mathrm{m}_{\mathrm{i}}$ is defined as:

$$
\hat{m}_{i}=\frac{(-1)^{i}}{(i) !} \int_{0}^{\infty} t^{i} h(t) d t
$$

is equal to the $\mathrm{i}^{\text {th }}$ time moment of $\left.\mathrm{h}(\mathrm{t})\right)$, multiplied by a constant factor. Note that the terms mi can be computed recursively in from (6) and (9), the transfer function $\mathrm{H}$ (s) can be expressed as:

$$
H(s)=\hat{m}_{o}+\hat{m}_{1} s+\hat{m}_{2} s^{2}+\hat{m}_{3} s^{3}
$$

In a reduced-order model, especially one obtained by moment matching, the transfer function is approximated by the reduced order system of proper rational function of $\mathrm{s}$ having q-poles:

$$
\hat{H}(s)=\frac{n_{q-1} s^{q-1}+n_{q-2} s^{q-2}+\ldots \ldots \ldots . .+n_{0}}{s^{q}+d_{q-1} s^{q-1}+\ldots \ldots \ldots \ldots \ldots . . d_{1} s+d_{0}}
$$

Because there are $2 q$ unknowns in the reduced-order system, it is forced to correspond to the first $2 q$ terms of (5) by using Pade' approximation [1]. In other words, $2 q$ low-order moments are required to obtain the reduced-order system having $\mathrm{q}$ poles, yielding the following equality

$$
\hat{H}(s)=m_{0}+m_{1} s+\ldots \ldots \ldots \ldots \ldots . . .+m_{2 q-1} s^{2 q-1}
$$

Multiplying both sides of (12) by the denominator of the left-hand side yields a set of equations that can be solved for $2 q$ coefficients. After finding roots of the denominator of the reduced-order model, (11) can be expressed as a partial fraction expansion form given by

$$
\hat{H}(s)=\sum_{i=1}^{q} \frac{r_{i}}{s-p_{i}}
$$

Where $r_{i}$ is a residue of $\hat{H}(s)$ at the pole $p_{i}$. It is then straightforward to obtain the approximated impulse response $\hat{h}(t)$ from (13), computing moments and obtaining the reduced-order model as described above has limitations: a reduced-order model of a stable circuits may be unstable. 


\subsection{Estimation Method of Power Estimation in Interconnect}

In order to find the power consumption or energy dissipation of a particular resistor element in a linear circuit, we first obtain the reduced-order model of current flowing through the resistor, denoted by $\hat{j}(s)$ (with the corresponding time-domain function $\hat{j}(t)$ ). using a model order reduction techniques The approximate energy dissipated by $R_{i}$, denoted by $\hat{E}_{i}$ during time period is then given by $\mathrm{t}_{1}, \mathrm{t}_{2}$ is given by

$$
\hat{E}_{i}=R_{i} \int_{1}^{2} \hat{j}^{2}(t) d t
$$

We make $t_{1}$ the time origin and $t_{2}$ infinite time. Then $\hat{j}(t)$ ) will reach a steady state, provided that $\hat{j}(t)$ corresponds to the reducedorder model of an individual transition. This leads us to the improper integral

$$
\hat{E}_{i}=R_{i} \int_{0}^{\infty} \hat{j}^{2}(t) d t
$$

First, we derive a general relation between improper integration in the time-domain and algebraic computation in the $s$-plane, which is expressed by the following theorem.

THEOREM-1: If the Laplace transform of a time-domain signal $\mathrm{h}(\mathrm{t})$, denoted by $\mathrm{H}(\mathrm{s}))$, has q singularities in the left half of the s-plane, then

$$
\int_{0}^{\infty} h^{2}(t) d t=\sum_{i=1}^{q} \hat{r}_{i}
$$

where $\hat{r}_{i}$ is a residue of $\left.\left.\mathrm{H}(-\mathrm{s})\right) \mathrm{H}(\mathrm{s})\right)$ at the singularity of $\mathrm{H}(\mathrm{s})$.)

$$
\text { Proof:_Let } I=\int_{0}^{\infty} h^{2}(t) d t
$$

From the definition of the Laplace transform, we have

$$
I=\left[\int_{0}^{\infty} h^{2}(t) e^{-s t} d t\right]_{s=0}
$$

Since the Laplace transform of a product of two functions is equal to the convolution of the Laplace transforms of two functions, we find that

$$
\begin{aligned}
I & =\left[\frac{1}{2 \pi i} \lim _{T \rightarrow \infty} \int_{/-T i}^{T i+\gamma} H(s-\omega) H(\omega) d \omega\right]_{s=0} \\
& =\frac{1}{2 \pi i} \lim _{T \rightarrow \infty} \int_{/-T i}^{+T i} H(-\omega) H(\omega) d \omega
\end{aligned}
$$

where $\gamma$ is chosen solely by the condition that it is to the right of the singularities of $\mathrm{H}(\mathrm{s})())$, meaning that $\gamma$ can be chosen as any real number larger than or equal to 0 . So we set $\gamma=0$ and take the contour of integration as a semicircle of radius $T$ with the line $R(s)=\gamma$ as diameter and to the left of it and the line segment $R(s)=\gamma,-T \leq T(s) \leq T$ as shown in Figure 2. By taking $T$ sufficiently large, we can guarantee that only the singularities of $\mathrm{H}(\mathrm{s})$ fall inside the contour, because $\mathrm{H}(\mathrm{s})$ has singularities to the right of the s-plane. Then, by the Cauchy residue theorem, It reduces to the sum of residues of $\mathrm{H} \mathrm{(-s).} \mathrm{H}$ (s) at the singularities of $\mathrm{H}(\mathrm{s})$, this concludes the proof.

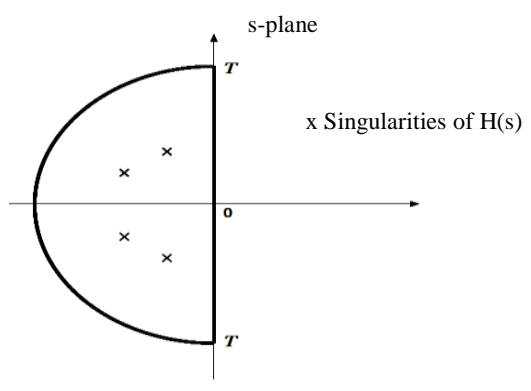

Figure-2. The singularities of $\mathbf{H}(\mathrm{s})$ and the contour of integration

THEOREM-2 If the Laplace transform of a time-domain signal $h(t)$, denoted by $H(s)$ ), has q simple poles in the left half of the s-plane, then

$$
\int_{0}^{\infty} h^{2}(t) d t=\sum_{i=1}^{q} r_{i} H\left(-p_{i}\right)
$$

Where $r_{i}$ is a residue of $\mathrm{H}(\mathrm{s})$ at the pole of $\mathrm{p}_{\mathrm{i}}$ of $\mathrm{H}(\mathrm{s})$.

Proof: From Theorem-1, the residue of $\mathrm{H}(-\mathrm{s}) \mathrm{H}(\mathrm{s})$ at the simple pole $\mathrm{p}_{\mathrm{i}}\left(\hat{r}_{i}\right)$ can be computed by

$$
\hat{r}_{i}=\lim _{s \rightarrow p_{i}}\left(s-p_{i}\right) H(s) H(-s)
$$

Because

$$
\lim _{s \rightarrow p_{i}}\left(s-p_{i}\right) H(s)=r_{i}
$$

We obtain the desired result from (16), (20) and (21):

$$
\int_{0}^{\infty} h^{2}(t) d t=\sum_{i=1}^{q} \hat{r}_{i}=\sum_{i=1}^{q} r_{i} H\left(-p_{i}\right)
$$

Notice that the relations derived in Theorems 1 and 2 are exact, rather than approximate. Thus, when the reduced-order model $\hat{H}(s))$ is used in (16) or (19), the accuracy of energy dissipation is determined by the accuracies of the poles and residues of the reduced-order model. The relations can also be used to derive the exact energy dissipation if we have the Laplace transform of the exact time-domain function of current.

\section{PROPOSED MODEL FOR ENERGY CALCULATION}

The focus of this paper is on the power distribution estimation of circuits consisting of lumped elements, we include the exact energy distribution of a distributed RLC interconnect for completeness. We consider a distributed RLC line as shown in figure.

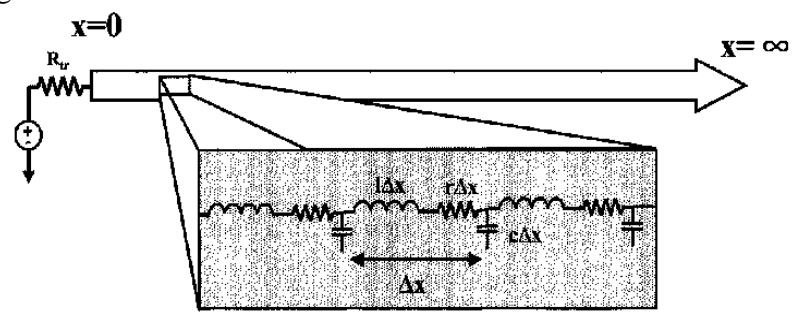

Figure-3 A distributed RLC Interconnect

Suppose that it is excited by a step input. Then, the Laplace transform of $v(x, t)$ for a distributed RLC line of infinite length is given by [12]

$$
V_{\text {out }}(x, s)=V_{\text {in }}(x, s) \cdot A \cdot B
$$


Where $A=\frac{Z_{0} \sqrt{\left(s+\frac{r}{l}\right) / s}}{Z_{0} \sqrt{\frac{\left(s+\frac{r}{l}\right)}{s}+R_{t r}}}$ and $\mathrm{B}=e^{-x \sqrt{l c} \sqrt{s\left(s+\frac{r}{l}\right)}}$

Where $Z_{0}=\sqrt{l / c}$ is the characteristic impedance of lossless transmission line, $r$ is the resistance per unit length, $\mathrm{c}$ is the capacitance per unit length, 1 is the inductance per unit length

$\mathrm{R}_{\mathrm{tr}}=Z_{s}$ is the driver resistance. For the step input the output equation is (by taking $R_{t r}=r$ )

$$
V_{\text {out }}(x, s)=\frac{\sqrt{l / c \sqrt{\left(s+\frac{r}{l}\right) / s}}}{s\left(\sqrt{l} / c \sqrt{\frac{\left(s+\frac{r}{l}\right)}{s}+r}\right)^{-x \sqrt{l c} \sqrt{s\left(s+\frac{r}{l}\right)}}}
$$

The current equation [10] in the time domain is given by

$$
\mathrm{I}(\mathrm{x}, \mathrm{t})=-\frac{1}{r} \frac{\partial v(x, t)}{\partial t}
$$

Table-1 Comparison of the energy distribution for

\begin{tabular}{|c|c|c|c|}
\hline \multicolumn{2}{|c|}{ Number of Nodes=1000 } & \multicolumn{2}{c|}{ Number of Nodes=1500 } \\
\hline $\begin{array}{c}\text { SPICE Model } \\
\text { (micro J) }\end{array}$ & $\begin{array}{c}\text { Our Model } \\
\text { (micro J) }\end{array}$ & $\begin{array}{c}\text { SPICE Model } \\
\text { (micro J) }\end{array}$ & $\begin{array}{c}\text { Our Model } \\
\text { (micro J) }\end{array}$ \\
\hline 0.2 & 0.2 & 0.2 & 0.2 \\
\hline 1 & 1 & 1 & 1 \\
\hline 8 & 8 & 8 & 8 \\
\hline 10 & 10 & 10 & 10 \\
\hline $10^{2}$ & 75 & $10^{2}$ & 85 \\
\hline $6 \times 10^{2}$ & 575 & $6 \times 10^{2}$ & 590 \\
\hline $8 \times 10^{2}$ & 700 & $8 \times 10^{2}$ & 795 \\
\hline $10^{3}$ & $8 \times 10^{2}$ & $10^{3}$ & $9.2 \times 10^{2}$ \\
\hline $10^{4}$ & $8.6 \times 10^{3}$ & $10^{4}$ & $9.25 \times 10^{3}$ \\
\hline $10^{5}$ & $8.5 \times 10^{4}$ & $10^{5}$ & $9.35 \times 10^{4}$ \\
\hline $10^{6}$ & $8.8 \times 10^{5}$ & $10^{6}$ & $9.57 \times 10^{5}$ \\
\hline $10^{7}$ & $9.5 \times 10^{6}$ & $10^{7}$ & $9.79 \times 10^{6}$ \\
\hline $10^{8}$ & $9.98 \times 10^{7}$ & $10^{8}$ & $9.98 \times 10^{7}$ \\
\hline
\end{tabular}

randomly generated RLC circuit

By applying Laplace transform on both side of (25)

$$
\begin{aligned}
I(x, s)=-\frac{1}{r}\left\{\frac{\sqrt{l / c} \sqrt{\left(s+\frac{r}{l}\right) / s}}{\left(\sqrt{l / c} \sqrt{\frac{\left(s+\frac{r}{l}\right)}{s}}+r\right)} e^{-x \sqrt{l c} \sqrt{s\left(s+\frac{r}{l}\right)}}\left(\sqrt{l c} \sqrt{\left(s\left(s+\frac{r}{l}\right)\right)}\right\}\right. \\
=\frac{l c\left(s+\frac{r}{l}\right) \mathrm{e}^{-\mathrm{x} \sqrt{l c} \sqrt{s\left(s+\frac{r}{l}\right)}}}{r s \sqrt{l c} \sqrt{(s+r / l) /}+r^{2} s}
\end{aligned}
$$

By equating the denominator term of (26) to zero, we get the pole of $\mathrm{I}(\mathrm{x}, \mathrm{s})$ as

$$
\mathrm{P}_{1}=0 \quad \& \quad P_{2}=-\frac{r}{l-c r^{2}}
$$

The pole $\mathrm{P}_{2}$ is in the left half of the s-plane, so we can use the relation given in Theorem-2. Now from (26) and (27)

$$
I\left(x,-p_{2}\right)=\frac{c\left(2 l-c r^{2}\right)}{\left(r^{2}+\sqrt{c\left(2 l-c r^{2}\right)}\right)} e^{-x \frac{r \sqrt{c\left(2 l-c r^{2}\right)}}{l-c r^{2}}}
$$

The residue of $\mathrm{I}(\mathrm{x}, \mathrm{s})$ at pole $\mathrm{P}_{2}$ is given by

$$
\hat{r}_{2}=\lim _{s \rightarrow p_{2}}\left(s-p_{2}\right) I(x, s)=r c^{2} e^{-x \frac{r \sqrt{r c}}{l-c r^{2}}}
$$

Thus the energy dissipation at the arbitrary position is given by from Theorem-2

$$
\begin{gathered}
E(x)=r \times \text { residue } \times I\left(x,-p_{2}\right) \\
=r \times r c^{2} \cdot e^{-x \frac{r \sqrt{r c}}{l-c r^{2}}} \times \frac{c\left(2 l-c r^{2}\right)}{\left(l-c r^{2}\right)\left(r^{2}+\sqrt{c\left(2 l-c r^{2}\right)} e^{-x \frac{r \sqrt{c\left(2 l-c r^{2}\right)}}{l-c r^{2}}}\right.} \\
=r^{2} c^{3} \times \frac{c\left(2 l-c r^{2}\right)}{\left(r^{2}+\sqrt{c\left(2 l-c r^{2}\right)}\right)} e^{-x \frac{r\left(\sqrt{c\left(2 l-c r^{2}\right)}+\sqrt{r c}\right)}{l-c r^{2}}}
\end{gathered}
$$

This is our approximated formula for the Energy dissipation in the Distributed RLC interconnects using model order reduction technique The obtained expression for the distribution of energy dissipation can be plotted as an exponential form as shown in the figure-4.

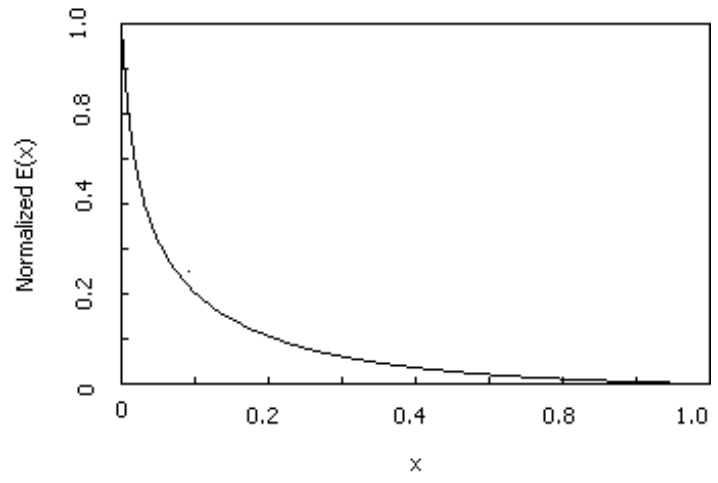

\section{Figure-4 The Distribution of Energy Dissipation for a distributed RLC Interconnect}

\section{RESULTS}

We have implemented the proposed power estimation method using Model Order Reduction technique and applied it to widely used actual interconnect RLC networks as shown in Figure-5. For each RLC network source we put a driver, where the driver is a step voltage source followed by a resister. Here power consumption and energy dissipation are used interchangeably.

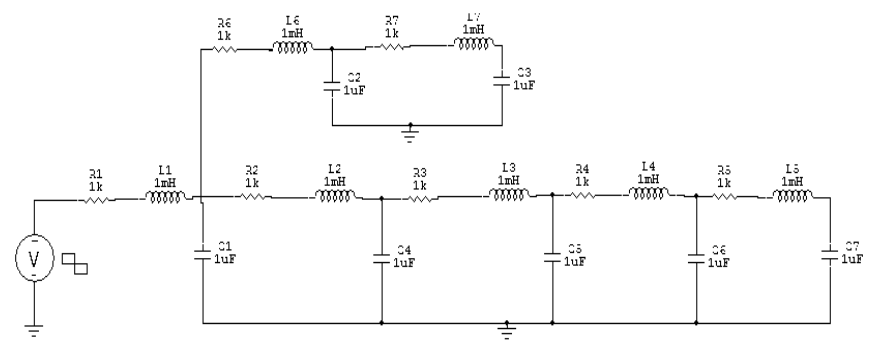

Figure-5 An RLC Tree Example

In order to verify the validity of the proposed analysis method, we randomly generate RLC Tree network while varying the number of nodes from 1000 to 1500 .We generate resistance, capacitance 
and inductance values in such way that the resulting circuit has widely varying time-constant and compare the energy distribution obtained by SPICE with that obtained by our methods. Table-1 gives the comparative result of the energy dissipation computed using SPICE and our method.

Figure-6 \& Figure-7 show the graphical representation of the result for the circuit with 1000 and 1500 nodes respectively. The result shows that a two pole approximation is quite accurate for most of the cases.

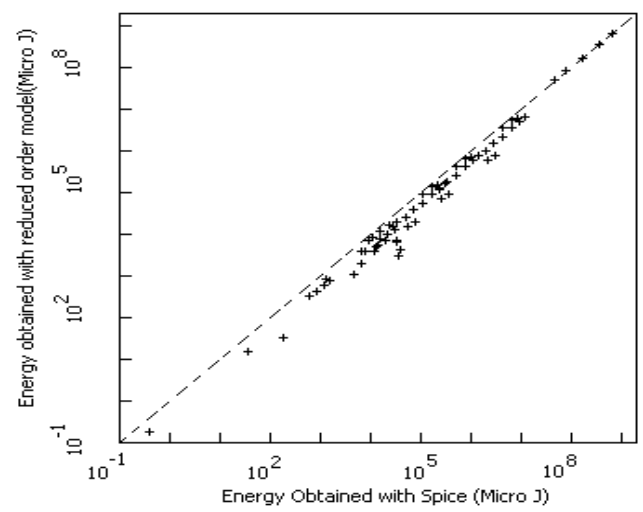

Figure-6 Comparison of the energy distribution for randomly generated RLC circuit with 1000 nodes

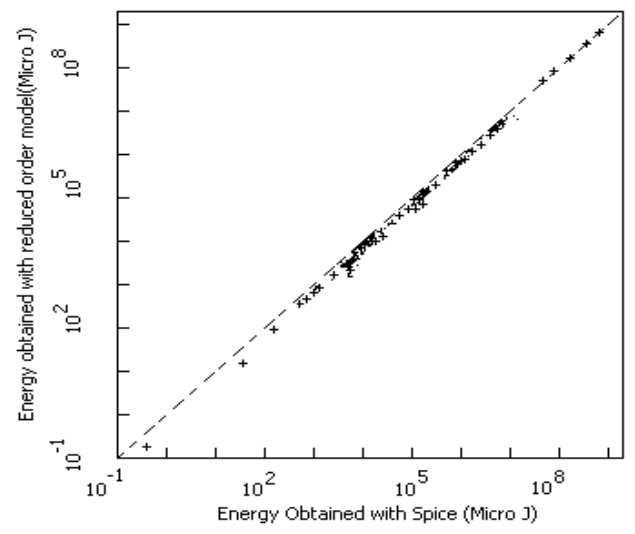

Figure-7 Comparison of the energy distribution for randomly generated RLC circuit with 1500 nodes.

\section{CONCLUSION}

We propose a method for the power estimation of RLC interconnects based on the reduced-order model. We show that power consumption can be computed efficiently in the s-domain using an algebraic formulation, instead of the improper integration in the time domain. The proposed method of computing power consumption relies on the poles and residues of the transfer function and can be used in any kind of model order reduction technique. Compact expressions that describe the energy distribution of a single distributed RLC interconnect are rigorously derived. Simple closed form approximation is derived that estimates the energy dissipation of semi-infinite distributed RLC interconnects.

\section{REFERENCES}

[1] Celik Mustafa, Lawrence Pileggi, Alten Odabasioglu, IC Interconnect Analysis, Kluwer Academic Publishers, 2002

[2] Shien-Yang Wu, Boon-Khim Liew, Young K.L, Yu C.H, and S.C. Analysis of Interconnect Delay for $0.18 \mu \mathrm{m}$ Technology and Beyond,. Interconnect Technology,. IEEE International Conference-1999. Pages: $68-70$.

[3] Lee K, On-chip interconnects - Giga Hertz and beyond, in Proc. Int. Interconnect Technology Conf., 1998, pp. 15-17.

[4] Deutsch, A., et al, When are Transmission-line effects important for on-chip interconnections? , IEEE Transactions on MTT, Oct. 1997, pp .1836-1847.

[5] Qi X, Kleveland B, Yu Z, Wong S.S, Dutton R.W, and Young T, On-chip inductance modeling of VLSI interconnects, in 2000 IEEE Int. Solid-State Circuits Conf. Dig. Techn. Papers, 2000, pp. 172-73

[6] Banerjee K, Mehrotra A, Sangiovanni-Vincentelli A, and $\mathrm{Hu}$ A, On thermal effects in deep sub-micron VLSI interconnects, in Proc. Design Automat. Conf., June 1999, pp. 885-891

[7] Shin Y, Sakurai T: Power Distribution Analysis of VLSI Interconnects Using Model Order Reduction. IEEE Tran. Computer Aided Design, Vol 21, pp. 739-745, June 2002

[8] Ismail Y.I, Friedman E.G and Neves J.L, Equivalent Elmore delay for RLC trees,. Proceedings of the $36^{\text {th }}$ ACM/IEEE conference on Design automation conference, 1999 pp. 715 720

[9] Pillage L.T and Rohrer R.A, Asymptotic Waveform Evaluation for timing analysis, IEEE Transactions on Computer-Aided Design, Vol. CAD-9, No. 4, pp. 352 - 366, April 1990.

[10] Shin Youngsoo, Sakurai T, Estimation of power distribution in VLSI interconnects,. Low Power Electronics and Design, International Symposium on, 2001. On page(s): 370-375.

[11] Cheng C.K, Lillis J, Lin S, and Chang N, Interconnect Analysis and Synthesis, John Wiley \& Sons, Inc., 2000.

[12] Davis J.A and Meindl J.D, Compact distributed RLC interconnect models - Part I: Single line transient, time delay and overshoot expressions, IEEE Trans. Electron Devices, vol. 47, pp. 2068-2077, Nov.2000. 